\title{
Influence of Light on Grape Berry Growth and Composition Varies during Fruit Development
}

\author{
N.K. Dokoozlian \\ Department of Viticulture and Enology, University of California, Davis, University of California, Kearney \\ Agricultural Center, 9240 South Riverbend Avenue, Parlier, CA 93648 \\ W.M. Kliewer \\ Department of Viticulture and Enology, University of California, Davis, CA 95616
}

Additional index words. Vitis vinifera, fruit growth, fruit composition

\begin{abstract}
Potted 'Cabernet Sauvignon' and 'Pinot noir' grapevines (Vitis vinifera L.) grown in a sunlit phytotron were used to study the influence of cluster light exposure during various stages of fruit development on berry growth and composition. Clusters grown without light during berry development stages I and II, stage III, or stages I, II, and III, were compared to clusters exposed to light throughout fruit development (control). The temperature of light-exposed and nonexposed fruit was similar. The weights and diameters of berries grown without light during stages I and II, or stages I, II, and III, were similar and significantly lower than those of the control. Fruit softening in both cultivars, as well as the initiation of berry coloration, was delayed when berries were grown without light during stages I and II. Following fruit softening, berries grown without light during stages I, II, and III were lower in sugar than the control. On the final sample date, 'Cabernet Sauvignon' berries grown without light during stages I, II, and III were higher in malate compared to the control. 'Pinot noir' berries grown without light during stages I and II, or stages I, II, and III, were lower in malate before fruit softening, and higher in malate following fruit softening, than the control. Control berries had greater skin anthocyanins and phenolics compared to the remaining treatments. Berries grown without light during stages I and II, or stage III, were greater in anthocyanins and phenolics than fruit grown without light during stages I, II, and III. Light had no effect on fruit tartrate concentration or juice $\mathrm{pH}$. Light had its greatest impact on fruit development during the initial stages of berry growth. Berry growth was reduced and ripening delayed when fruit were grown without light during stages I and II. Normal fruit development was not fully restored when these fruit were exposed to light during stage III.
\end{abstract}

Grape berry development may be divided into two major stages of growth, separated by a lag phase (Coombe, 1976). During stage I berry pericarp growth is rapid, at first due to cell division and expansion, and later due to cell expansion alone (Harris et al., 1968). Berries accumulate organic acids but little sugar during stage I, and remain green and hard. Stage II is referred to as the lag phase of development, as berry growth slows. Rapid berry growth, as a result of cell enlargement, resumes with the initiation of stage III. During stage III sugar and color accumulate rapidly, and the concentration of organic acids declines.

Light influences the growth and composition of a wide variety of fruit, including grapes. Grape berries exposed to sunlight are generally higher in sugars, anthocyanins, and phenolics, and lower in titratable acidity, malate, and $\mathrm{pH}$, compared to berries ripened in canopy shade (Kliewer, 1977; Kliewer and Antcliff, 1970; Kliewer and Lider, 1968; Morrison, 1988; Reynolds et al., 1986). Previous studies have shown that the effects of temperature (Hale and Buttrose, 1974) and vine water status (Hardie and Considine, 1976) on berry size and composition vary during fruit ontogeny. The purpose of this investigation was to determine the influence of cluster light exposure during specific stages of berry development on fruit growth and composition.

Received for publication 27 Oct. 1995. Accepted for publication 15 Mar. 1996. 1996. We thank B.A. Bonner, L.E. Williams, and R.M. Pool for their helpful reviews of this manuscript. Financial support was provided by the American Vineyard Foundation and the Dept. of Viticulture and Enology, Univ. of California, Davis. The cost of publishing this paper was defrayed in part by the payment of page charges. Under postal regulations, this paper therefore must be hereby marked advertisement solely to indicate this fact.

\section{Materials and Methods}

Plant materials and growth conditions. Three-year-old own rooted grapevines of Vitis vinifera L. cultivars 'Cabernet Sauvignon' and 'Pinot noir' were used in the study. Plants were grown in 12L plastic pots containing 1 soil : 1 sand : 1 peat medium (by volume). Vines were grown outdoors under full sunlight for two seasons before their use. At the initiation of the experiment, 22 dormant vines of each cultivar were pruned to four, two-bud spurs and placed in the stationary phytotron at the Univ. of California, Davis. The phytotron is illuminated with natural sunlight, and provided a diurnal temperature range between $28 \pm 2.4^{\circ} \mathrm{C}$ (day) and $16 \pm 1.8{ }^{\circ} \mathrm{C}$ (night). Relative humidity was maintained at $40 \%$. Shoot growth was initiated $10 \mathrm{~d}$ after the vines were placed in the phytotron. When shoot length was about $0.3 \mathrm{~m}$, each vine was thinned to four cluster bearing shoots. Shoots were vertically trained on bamboo stakes anchored in the soil of the pot.

Treatments and experimental design. Anthesis occurred about one month after budbreak. At fruit set, 16 vines of each cultivar were selected on the basis of uniformity of shoot growth and cluster development. Extra vines were removed from the phytotron. Shoots were thinned to one cluster, and four treatments (one per shoot) randomly assigned to each vine as follows:

1) Clusters exposed to light during stages I, II, and III (experimental control).

2) Clusters grown without light during stages I and II, and exposed to light during stage III.

3) Clusters exposed to light during stages I and II, and grown without light during stage III.

4) Clusters grown without light during stages I, II, and III. 

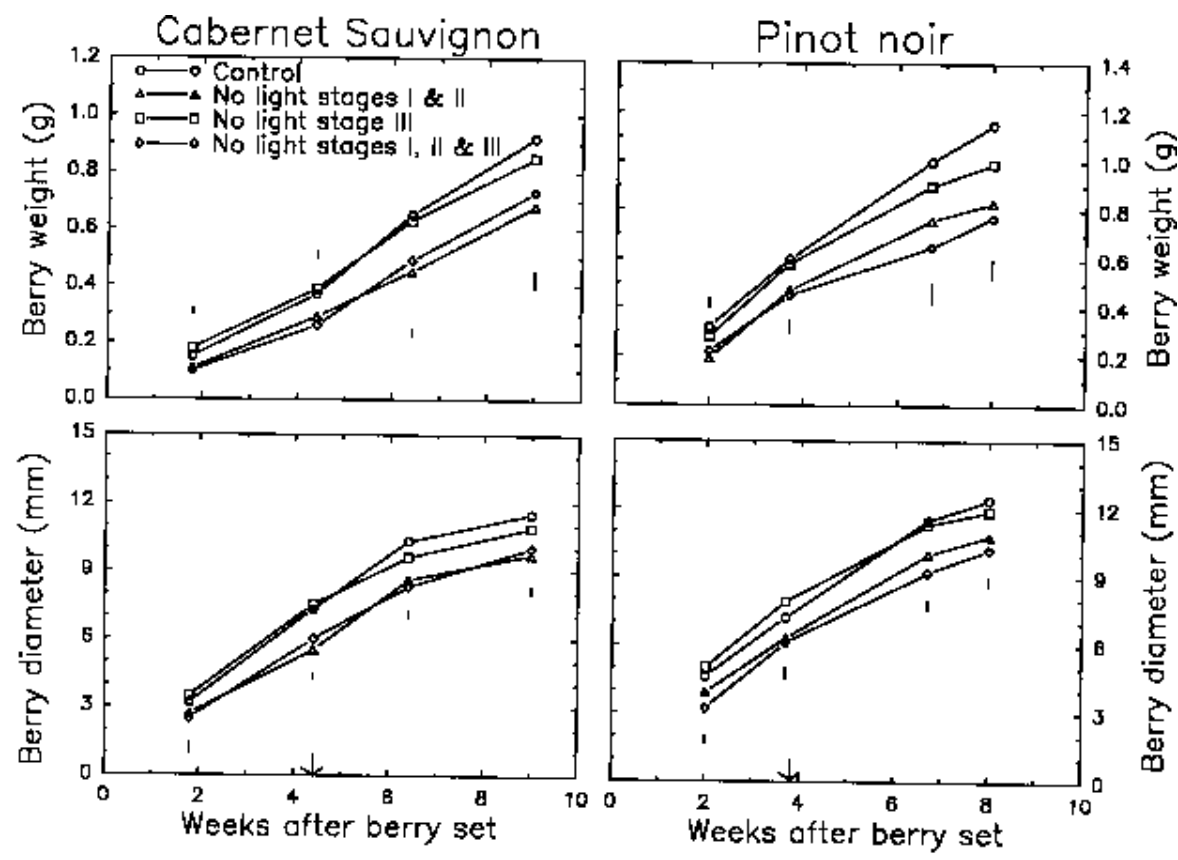

plant served as an experimental unit. The vines of each cultivar were arranged in a randomized complete-block design consisting of four, four vine blocks.

Clusters grown in the absence of sunlight were placed in $17 \times 20-\mathrm{cm}$ aluminum-coated, white paper bags. Basal leaves on all shoots were removed to facilitate the fastening of bags to the shoot; contact between the bags and clusters was prevented. To encourage ventilation, one end of an $18 \mathrm{~cm}$ long black plastic tube $(0.8 \mathrm{~cm}$ inner diameter) was inserted into each bag next to the cluster. Tubing outside the bag was wrapped in a ring to prevent light from entering the tube. The tops and bottoms of each bag were sealed tightly with black adhesive tape. Bags were opened briefly at night to apply fungicides.

A LI-190S quantum sensor attached to a LI-185 quantum meter (LI-COR, Lincoln, Neb.) was used to ensure that the interior of each bag was free of detectable light at the initiation of the experiment. A CR21 data-

Fig. 1. Influence of cluster light exposure during various stages of fruit development on the weight and diameter of 'Cabernet Sauvignon' (left column) and 'Pinot noir' (right column) grape berries. Arrows above the horizontal axis indicate the initiation of berry softening for each cultivar. Vertical lines represent the LSD $(P$ $\leq 0.05)$ of the treatment means for each sample date.

In this study fruit development stages I and II refer to the period between berry set ( 2 to $3 \mathrm{~mm}$ in diameter) and the initiation of berry softening, while stage III refers to the period between fruit softening and maturity. Each vine received all four treatments; thus, each

Fig. 2. Influence of cluster light exposure during various stages of fruit development on the glucose and fructose concentrations of 'Cabernet Sauvignon' (left column) and 'Pinot noir' (right column) grape berries. Arrows above the horizontal axis indicate the initiation of berry softening for each cultivar. Vertical lines represent the $\operatorname{LSD}(P \leq 0.05)$ of the treatment means for each sample date. ${ }^{\text {Ns Nonsignificant }}$ at the $P=0.05$. logger (Campbell Scientific, Logan, Utah) with four quantum sensors was used to monitor photosynthetically active radiation (PAR) incident to one control cluster in each experimental block. Quantum sensors were positioned horizontally, immediately above the clusters. PAR in this region was about $20 \%$ of ambient. Spectroradiometer measurements (LI-1800) revealed that the spectral composition of sunlight and light in the phytotron was similar. The temperature of light-exposed and nonexposed fruit was determined by inserting thermocouples into randomly selected berries. The berries were discarded after the 24 -h measurement period was completed. Measurements were taken on four sets of 'Cabernet Sauvignon' berries and two sets of 'Pinot noir' berries at various times throughout the experiment. The temperature of light-exposed and nonexposed fruit was similar, and exceeded air temperature by 1 to $3{ }^{\circ} \mathrm{C}$.

Clusters from one randomly selected vine in each block were harvested 14, 32, 46, and 63 ('Cabernet Sauvignon') or 14, 25, 42, and 56 ('Pinot noir') d after berry set. These dates coincided with the following stages of berry development for each cultivar: middle of stage I (harvest 1 ); $20 \%$ berry softening (harvest 2 ); middle of stage III (harvest 3); and the end of stage III (harvest 4). To maintain a constant light environment, harvested vines remained in the phytotron until the completion of the study.

Fruit analysis. Harvested clusters were placed in plastic bags and transported on ice to the laboratory. Berries were removed from the rachis, weighed, and their diameters recorded with a caliper. On the second sample date the number of softened berries (i.e., berries with compressible flesh), and the number of berries with $25 \%$ or more of their surface colored, were recorded. Berries were then randomly separated into two equal subsamples. One sample was used for $\mathrm{pH}$, sugar, and acid determinations, and the other for anthocyanin and total phenolic determinations. The samples were stored in sealed plastic bags at $-20^{\circ} \mathrm{C}$ 
until analyzed. Sample size was about 20 to 25 berries for 'Cabernet Sauvignon', and 25 to 30 berries for 'Pinot noir'.

Frozen berries were thawed at room temperature in $250-\mathrm{mL}$ breakers. Berries were crushed and skins macerated with a pestle. Juice $\mathrm{pH}$ was determined after $30 \mathrm{~min}$ of settling. Following $\mathrm{pH}$ determinations, juice samples were quantitatively transferred to their original beaker. Beakers were covered with a watch glass and boiled for $30 \mathrm{~min}$ with frequent stirring. Samples were cooled and filtered through Whatman no. 2 filter paper into a $250-\mathrm{mL}$ volumetric flask. Following two $25-\mathrm{mL}$ rinses with boiling distilled water, the filter paper and solid material were discarded, the filtrate was brought to volume with distilled water. An aliquot of this solution was retained for sugar and acid analyses. The hexose sugar and organic acid fractions of the samples were determined by high-performance liquid chromatography [pump model 1330, differential refractometer model 1770, and organic acid analysis column HPX-87H $(300 \times 7.8 \mathrm{~mm})$, Bio-Rad Laboratories, Richmond, Calif.] according to the procedure of McCord et al. (1984).

Skin discs (4 $\mathrm{mm}$ in diameter) were removed from the equator of 20 frozen berries in each sample. The discs were placed in a polystyrene tube containing $50 \mathrm{~mL}$ of acidified methanol $(1 \% \mathrm{HCl}$ $\mathrm{v} / \mathrm{v})$ and extracted in darkness. After $48 \mathrm{~h}$, the samples were mixed and allowed to settle. Absorbance was determined at $520 \mathrm{~nm}$. Anthocyanin concentration (anthocyanin $/ \mathrm{cm}^{2}$ of berry skin) was determined using a molar absorbance of 28,000 and a molecular weight of 529 (Amerine and Ough, 1980). Total phenolics were determined on an aliquot of the above extract using the automated method of Slinkard and Singleton (1977), and were expressed as mg gallic acid equivalents $(\mathrm{GAE}) / \mathrm{cm}^{2}$ berry skin.

\section{Results}

Berry growth. Differences in berry size among the treatments in both cultivars were established before berry softening, and generally maintained throughout ripening (Fig. 1). The weight and diameter of berries grown without light during stages I and II, or stages I, II, and III, were similar and significantly lower compared to the control. In contrast, the growth of control berries and berries grown without light during stage III were similar. An exception was noted for 'Pinot noir' on the final sample date, as the weight of berries grown without light during stage III was lower compared to the control.

Berry composition. Berry hexose sugar concentrations were similar among the treatments in both cultivars following fruit set, with total sugar concentration (glucose + fructose) ranging between 10 to $15 \mathrm{mg} \cdot \mathrm{g}^{-1}$ fresh weight (Fig. 2). Berry softening was delayed when clusters were grown without light during stages I and II (Table 1). Following fruit softening, however, the sugar concentrations of all treatments increased sharply. Immediately after fruit softening, control fruit of 'Cabernet Sauvignon' had greater hex- ose sugar concentrations than the remaining treatments. On the final sample date, 'Cabernet Sauvignon' control berries and berries grown without light during stages I and II had similar hexose sugar concentrations. Berries of this cultivar grown without light during stages I, II, and III, as well as those grown without light during stage III, were lower in sugar than the control on the final sample date. Following fruit softening in 'Pinot noir', berries grown without light during stages I, II, and III were lower than the control, while the sugar concentrations of berries grown without light during stages I and II, or stage III, were similar to the control.

Following berry set, the malate concentration of the control and berries grown without light during stages I and II were similar for 'Cabernet Sauvignon' (Fig. 3). After berry softening in this cultivar, malate declined more rapidly in the control than in the other treatments. On the final sample date, however, only berries grown without light during stages I, II, and III were greater in malate than the control. Berries of 'Pinot noir' grown without light during stages I and II were lower in malate following berry set, and at berry softening, compared to the control. Immediately following fruit softening in 'Pinot noir', berries grown without light during stages I and II, or stage III, were greater in malate than the control. On the final sample date, control berries of this cultivar were lower in malate compared to the remaining treatments. Cluster light exposure had no effect on the berry tartrate concentration or juice $\mathrm{pH}$ of either cultivar (Fig. 3).

Color initiation was delayed in both cultivars when berries were grown without light during stages I and II (Table 1). In both cultivars, treatments grown without light during stages I and II were lower in anthocyanins compared to the control (Fig. 4). On the final harvest date in both cultivars, anthocyanins were greatest for control berries and lowest for berries grown without light during stages I, II, and III. Berries grown without light during stage III were also lower in anthocyanins than the control, but had more pigment than berries grown without light during stages I, II, and III. Treatment differences in phenolics followed the same relationship as described for anthocyanins (Fig. 4). In both cultivars, phenolics were greatest for control berries and lowest for berries grown without light during stages I, II, and III.

\section{Discussion}

The temperature of grape berries is directly related to their incident radiation (Smart and Sinclair, 1976); thus, daytime temperatures of sunlight-exposed berries may be $11^{\circ} \mathrm{C}$ or more greater than nonexposed berries, depending on time of day and solar conditions (Kliewer and Lider, 1968; Reynolds et al., 1986). It is therefore difficult to separate the effects of light and temperature on fruit development in the field. In the present study, berry temperatures among the treatments were similar. Reported differences in berry growth and composition are therefore attributed to

Table 1. Influence of cluster light exposure on the softening and coloration of 'Cabernet Sauvignon' and 'Pinot noir' grapes. ${ }^{\mathrm{Z}}$

\begin{tabular}{|c|c|c|c|c|}
\hline Cultivar & $\begin{array}{c}\text { Days after } \\
\text { berry set }\end{array}$ & Treatment & $\begin{array}{c}\text { Softened } \\
\text { berries (\%) }\end{array}$ & $\begin{array}{c}\text { Colored } \\
\text { berries }(\%)\end{array}$ \\
\hline \multirow{2}{*}{ Cabernet Sauvignon } & 31 & Control & $32 \mathrm{a}^{\mathrm{y}}$ & $18 \mathrm{a}$ \\
\hline & & No light stages I and II & $4 \mathrm{~b}$ & $0 \mathrm{~b}$ \\
\hline \multirow[t]{2}{*}{ Pinot noir } & 26 & Control & $25 \mathrm{a}$ & $18 \mathrm{a}$ \\
\hline & & No light stages I and II & $6 \mathrm{~b}$ & $0 \mathrm{~b}$ \\
\hline
\end{tabular}

${ }^{\mathrm{z}}$ Control clusters received about $20 \%$ of ambient photosynthetically active radiation. Clusters grown without light were placed in aluminum foil bags at berry set.

'Means for each cultivar followed by different letters within columns are significantly different at $P=0.05$ (Duncan's multiplerange test). 


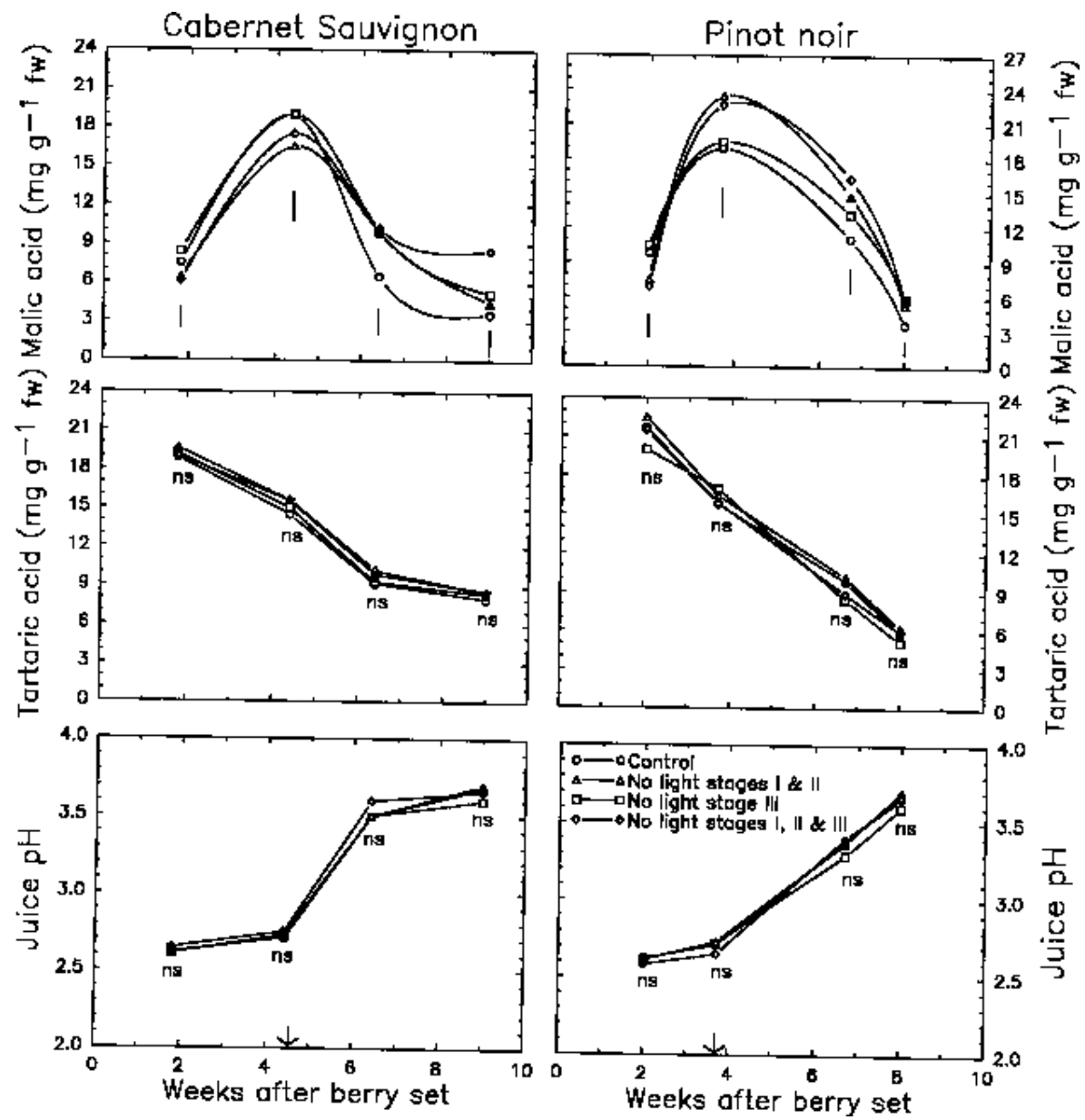

Fig. 3. Influence of cluster light exposure during various stages of fruit development on the malate and tartrate concentrations, and juice $\mathrm{pH}$, of 'Cabernet Sauvignon' (left column) and 'Pinot noir' (right column) grape berries. Arrows above the horizontal axis indicate the initiation of berry softening for each cultivar. Vertical lines represent tie LSD $(P \leq 0.05)$ of the treatment means for each sample date.

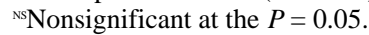

the direct effects of light, rather than differences in fruit temperature as a result of cluster light exposure.

Berries grown without light during the initial stages of fruit development had lower weights and diameters than berries exposed to light during this same period. These results are contrary to previous studies, which reported that sunlight-exposed berries were smaller than berries grown in paper bags or canopy shade (Crippen and Morrison, 1986; Kliewer and Lider, 1968; Reynolds et al., 1986). Reynolds et al. (1986) suggested that the higher berry temperatures associated with sunlight exposure may inhibit fruit growth. The optimum temperature for grape berry growth ranges between 25 to $30^{\circ} \mathrm{C}$ (Hale and Buttrose, 1974). Kliewer and Lider (1968) and Reynolds et al. (1986) reported the daytime temperature of sunlight-exposed berries often exceeds $30^{\circ} \mathrm{C}$, while the temperature of nonexposed berries is maintained within the optimum range for growth. The transpiration rate of sunlight-exposed fruit is also greater compared to nonexposed fruit (Blanke and Leyhe, 1987), and several investigators have associated the decreased size and higher soluble solids of sunlight-exposed fruit to their lower turgor and higher transpiration rates (Crippen and Morrison, 1986; Reynolds et al., 1986). Plants used in this experiment were well watered at all times, thus diurnal or seasonal fluctuations in vine water status were negligible.

During the initial stages of development, grape pericarp growth is a result of cell division and expansion (Harris et al., 1968). Light has been reported to activate cell division and induce cell expansion in leaves of Phaseolus vulgaris L. (Humphries and Wheeler, 1960; Verbelen and De Greef, 1979). Blanpied and Wilde (1968) reported that cell division in nonexposed (bagged) apple fruit was lower than in sunlight-exposed fruit shortly after fruit set. The authors concluded that these differences were at least partially due to the lower temperature of nonexposed fruit. In the current experiment, differences in berry size between light-exposed and nonexposed fruit appear to be due to light-mediated effects on cell division and/or cell enlargement, particularly during the initial stages of growth. While berries grown in the absence of light during stages I and II were significantly smaller than the control, berries grown without light during stage III were similar in size ('Pinot noir'), or only slightly smaller ('Cabernet Sauvignon'), compared to the control. In addition, berries grown without light during stages I and II, and exposed to light during stage III, were similar in weight and diameter to berries grown without light during stages I, II, and III. Thus, reductions in berry size resulting from sunlight exclusion during stages I and II could not be reversed via subsequent light exposure in stage III.

Several mechanisms may be responsible for the photocontrol of berry growth, including light-mediated effects on fruit photosynthesis and carbon metabolism. Based on published assimilation rates (Kriedemann, 1968), fruit photosynthesis contributed $<1 \%$ of the estimated dry weight difference between light-exposed and nonexposed fruit in this study. Hole and Scott (1981) suggested that, despite its small contribution to total fruit dry weight at maturity, fruit photosynthesis during the initial stages of growth may be critical to subsequent fruit development. Light may also be essential for regulating the importation or metabolism of carbon and other assimilates in young fruit. Schapendonk and Brouwer (1984) reported a 50\% reduction in the accumulated dry weight of cucumber fruit as a result of fruit shading. These authors estimated that fruit photosynthesis contributed only $2 \%$ to $7 \%$ of the total dry weight in sunlight-exposed fruit, and concluded that the elimination of fruit photosynthesis was not fully responsible for the dry weight differences between light-exposed and shaded fruit. They suggested that the sink strength of shaded fruit was reduced, and that sink function may be partially regulated by a growth factor synthesized in the light. Hole and Scott (1981) reported that the yield of pea was significantly reduced by fruit shading, but concluded that only a small portion of this reduction could be attributed to an absence of fruit photosynthesis. Their results also suggest that shading young fruit may permanently reduce sink strength. Light may also influence the concentration or activity of phytohormones which regulate fruit growth and development (Coombe, 1973).

The results of this study are in agreement with previous reports that sugar accumulation is greater for light-exposed fruit than for nonexposed fruit (Brown and Coombe, 1985; Crippen and Morrison, 1986; Kliewer and Lider, 1968; Reynolds et al., 1986). Compared to the control fruit softening was delayed, and sugar accumulation 


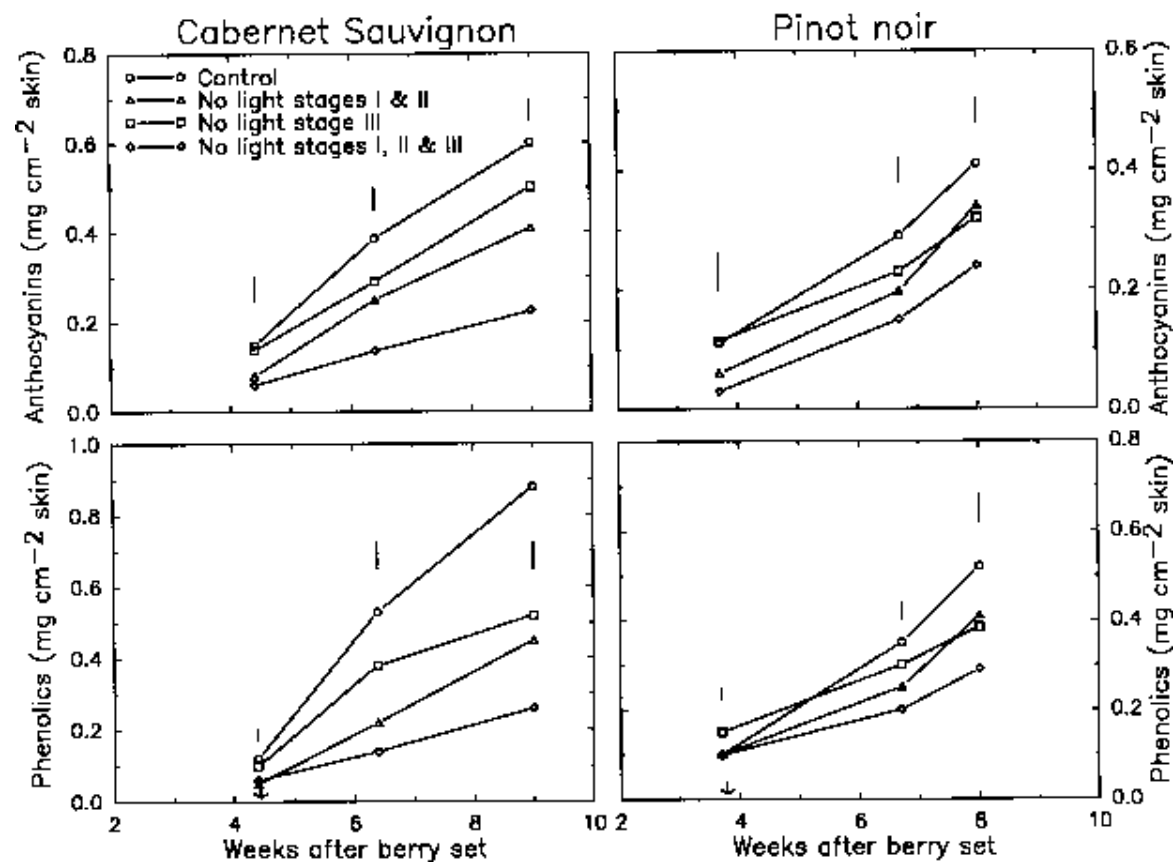

results of the current study suggest that the effects of light on berry $\mathrm{pH}$ may be indirect, and perhaps related to the concomitant effects on berry temperature. The results also lend support to the suggestion that, with regard to juice $\mathrm{pH}$, foliage light exposure is more important than cluster light exposure (Morrison, 1988).

Light-exposed fruit are generally higher in anthocyanins and phenolics compared to nonexposed fruit (Kliewer, 1977; Kataoka et al., 1984). Roubelakis-Angelakis and Kliewer (1986) found that the activity of phenylalanine ammonia-lyase, a key enzyme in secondary metabolism, increased when grape berries were exposed to light. Although grape berries do not accumulate pigments during this period, light exposure during stages I and II appears necessary for maximum pigment production during stage III. Exposing fruit to light before the onset of pigment production may increase the initial concentration or activity of one or several anthocyanin biosynthetic enzymes (Takeda et al., 1988). Once pigment accumu-

Fig. 4. Influence of cluster light exposure during various stages of fruit development on the anthocyanin and total phenolic concentration of 'Cabernet Sauvignon' (left column) and 'Pinot noir' (right column) grape berries. Arrows above the horizontal axis indicate the initiation of berry softening for each cultivar. Vertical lines represent the LSD $(P \leq 0.05)$ of the treatment means for each sample date. Ns Nonsignificant at the $P=0.05$.

reduced, when fruit were grown without light during stages I, II, and III. Based on the available data it is unclear whether the lower sugar content of these fruit resulted from a delay in berry softening, or if a reduction in the rate of glucose and fructose accumulation following fruit softening also occurred. The activity of at least one enzyme regulating sugar metabolism, invertase, is believed to be photoregulated in grape berries (Kliewer and Smart, 1989). A reduction in the concentration or activity of invertase, or other enzymes responsible for the regulation of sugar accumulation, may have been responsible for the lower sugar concentration of nonexposed fruit. Sugar accumulation is also inhibited when berry growth during stage III is restricted (Coombe, 1973). Perhaps due to their smaller size, or to alterations in their pericarp cell walls, the ability of berries grown without light during stages I, II, and III to expand and accumulate solutes during ripening may have been limited.

Under field conditions, light-exposed fruit are generally lower in malate than nonexposed fruit (Crippen and Morrison, 1986; Reynolds et al., 1986). These differences are normally attributed to the higher temperatures and respiration rates of light-exposed fruit (Kliewer and Lider, 1968; Lakso and Kliewer, 1978). In this study, light-exposed 'Pinot noir' berries had higher malate levels before fruit softening, and lower malate levels following fruit softening, compared to nonexposed berries. Since light is not known to influence malic acid accumulation in grape tissues (Ruffner, 1982), differences in malate accumulation and degradation in this study were likely due to treatment effects on fruit maturation rate.

Light had no effect on fruit tartaric acid concentration or juice $\mathrm{pH}$ in this study. That fruit tartaric acid concentration was not affected by light is in agreement with previous reports (Crippen and Morrison, 1986; Kliewer and Lider, 1968). However, field investigations indicated that sunlight-exposed fruit had significantly lower juice pH than nonexposed fruit (Smart, 1985). The lation is initiated, light is needed to maintain the maximum activity of these enzymes during ripening.

\section{Literature Cited}

Amerine, M.A. and C.S. Ough. 1980. Methods for analysis of musts and wines. Wiley and Sons, New York.

Blanke, M.M. and A. Leyhe. 1987. Stomatal activity of the grape berry cv. 'Riesling', 'Muller-Thurgau' and 'Ehrenfelser'. J. Plant Physiol. 127:451460.

Blanpied, G.D. and M.H. Wilde. 1968. A study of the cells in the outer flesh of developing 'McIntosh' apple fruits. Bot. Gaz. 129:173-183.

Brown, S.C., and B.G. Coombe. 1985. Solute accumulation by grape pericarp cells. III. Sugar changes in vivo and the effect of shading. Biochem. Physiol. Pflanzen 180:371-381.

Coombe, B.G. 1973. The regulation of set and development of the grape berry. Acta Hort. 34:261-274.

Coombe, B.G. 1976. The development of fleshy fruits. Annu. Rev. Plant Physiol. 27:507-528.

Crippen, D.D., and J.C. Morrison. 1986. The effects of sun exposure on the compositional development of 'Cabernet Sauvignon' berries. Amer. J. Enol. Viticult. 37:235-242.

Hale, C.R. and M.S. Buttrose. 1974. Effect of temperature on ontogeny of berries of Vitis vinifera L. cv. 'Cabernet Sauvignon'. J. Amer. Soc. Hort. Sci. 99:390-394.

Hardie, W.J. and J.A. Considine. 1976. Response of grapes to waterdeficit stress in particular stages of development. Amer. J. Enol. Viticult. 27:55-61.

Harris, J.M., P.E. Kriedemann, and J.V. Possingham. 1968. Anatomical aspects of grape berry development. Vitis 7:106-109.

Hole, C.C. and P.A. Scott. 1981. The effect of fruit shading on yield in Pisum sativum L. Ann. Bot. 48:827-835.

Humphries, E.C., and A.W. Wheeler. 1960. The effects of kinetin, gibberellic acid, and light on expansion and cell division in leaf disc of dwarf bean (Phaseolus vulgaris). J. Expt. Bot. 11:81-85.

Kataoka, I., Y. Kubo, A. Sugiura, and T. Tomana. 1984. Effects of temperature, cluster shading and some growth regulators on L-phenylalanine ammonia-lyase activity and anthocyanin accumulation in black grapes. Mem. Coll. Agr. Kyoto Univ. 124:35-44.

Kliewer, W.M. 1977. Influence of temperature, solar radiation, and nitrogen on coloration and composition of 'Emperor' grapes. Amer. J. Enol. Viticult. 28:96-103.

Kliewer, W.M. and A.J. Antcliff. 1970. Influence of defoliation, leaf 
darkening, and cluster shading on the growth and composition of 'Sultana' grapes. Amer. J. Enol. Viticult. 21:26-36.

Kliewer, W.M. and L.A. Lider. 1968. Influence of cluster exposure to the sun on the composition of 'Thompson Seedless' fruit. Amer. J. Enol. Viticult. 19:175-184.

Kliewer, W.M. and R.E. Smart. 1989. Canopy manipulation for optimizing vine microclimate, crop yield, and composition of grapes, p. 275291. In: C.J. Wright (ed.). Manipulation of Fruiting. Butterworth, London.

Kriedemann, P.E. 1968. Observations on gas exchange in the developing 'Sultana' berry. Austral. J. Biol. Sci. 21:907-916.

Lakso, A.N. and W.M. Kliewer. 1978. Theinfluence of temperature onmalic acid metabolism in grape berries. II. Temperature responses of net dark $\mathrm{CO}_{2}$ fixation and malic acid pools. Amer. J. Enol. Viticult. 29:145-149.

McCord, J.D., E. Trousdale, and D.D.Y. Ryu. 1984. An improved sample preparation procedure for the analysis of major organic components in grape must and wine by high performance liquid chromatography. Amer. J. Enol. Viticult. 35:28-29.

Morrison, J.C. 1988. The effects of shading on the composition of 'Cabernet Sauvignon' grape berries. Proc. 2nd Intl. Cool Climate Viticulture and Oenology. p. 144-146.

Reynolds, A.G., R.M. Pool, and L.R. Mattick. 1986. Influence of cluster exposure on fruit composition and wine quality of Seyval blanc grapes. Vitis 25:85-95.

Roubelakis-Angelakis, K.A. and W.M. Kliewer. 1986. Effects of exogenous factors on phenylalanine ammonia-lyase activity and accumulation of anthocyanins and total phenolics in grape berries. Amer. J. Enol. Viticult. 37:275-280.

Ruffner, H.P. 1982. Metabolism of tartaric and malic acids in Vitis: A review-Part B. Vitis 21:346-358.

Schapendonk, A.H.C.M. and P. Brouwer. 1984. Fruit growth of cucumber in relation to assimilate supply and sink activity. Sci. Hort. 23:21-33.

Slinkard, P., and V.L. Singleton. 1977. Colorimetry of total phenolics with phosphotungstic acid reagents. Amer. J. Enol. Viticult. 17:126-134.

Smart, R.E. 1985. Principles of grapevine canopy management microclimate manipulation with implications for yield and quality. A review. Amer. J. Enol. Viticult. 36:230-239.

Smart, R.E. and T.R. Sinclair. 1976. Solar heating of grape berries and other spherical fruits. Agr. Meteorol. 17:241-259.

Takeda, K., D. Fisher, and H. Grisebach. 1988. Anthocyanin composition of Sinapis alba, light induction of enzymes and biosynthesis. Phytochem. 27:1351-1353.

Verbelen, J.P. and J.A. DeGreef. 1979. Leaf development of Phaseolus vulgaris L. in light and in darkness. Amer. J. Bot. 66:970-976. 